\title{
Una Tipología de pequeños agricultores de las fincas de San Andrés De Tupicocha
}

\author{
A typology of smallholder farmers in San Andres De Tupicocha
}

\author{
Cecilia E. Figueroa S. ${ }^{1} \&$ María N. Varas C. ${ }^{2}$
}

\begin{abstract}
Resumen
El manejo del agua dentro de un sistema agrario es un factor importante para determinar la diversidad de estrategias con las que manejar la variedad de cultivos y conocer el status económico de los agricultores. El objetivo del presente trabajo fue desarrollar una tipología compuesta de familias agrícolas para poder entender y categorizar la diversidad de estrategias de subsistencia entre los minifundistas del distrito de San Andrés de Tupicocha. Para este fin, analizamos con frecuencias la tipología en términos de su capacidad para distinguir patrones en el manejo de los recursos, como los cultivos y el agua. También evaluamos datos socioeconómicos a partir de encuestas aplicadas a las familias campesinas y diagnósticos participativos de agricultores, el reunir a las familias en grupos homogéneos y significativos y el estudio de la variabilidad en el manejo del agua dentro de cada agrupación. Encontramos cinco clases de agricultores con comportamientos desde un control masivo de las fuentes de agua y de la diversidad de cultivos hasta el control mínimo de los recursos, pero que han mostrado tener estrategias complementarias para la supervivencia como son la venta de la mano de obra y el desarrollo de las actividades no agropecuarias.
\end{abstract}

Palabras clave: agua; diversidad; sistema; tipología.

\begin{abstract}
Managing water within an agricultural system is an important factor to determine the diversity of strategies to manage crop diversity and get to know the economic status of farmers. The objective of this research was to develop a typology consisting of agricultural families to understand and categorize the diversity of livelihood strategies among smallholders in the district of San Andrés de Tupicocha. To this end, we analyzed the type frequencies in terms of their ability to distinguish patterns in the management of resources such as crops and water. We also evaluated socio-economic management data using surveys of farm families and farmers participatory diagnosis, bringing families together into homogeneous and significant groups, and the study of variability in water management within each group. We found five types of farmers with behaviors ranging from massive control of water sources and crop diversity to minimal control of resources, but have shown to have complementary strategies for survival, such as the sale of labor and the development of non-agricultural activities. Keywords: water; diversity; system; typology.
\end{abstract}

\section{Introducción}

Las tipologías son una manera de representar la diversidad de sistemas agrícolas y las unidades de producción en una región dada (Aguirre, 1990; Valbuena et al., 2008). A partir de la tipología, se pueden identificar grupos de trabajo homogéneos en cuanto a objetivos e intereses y para los cuales se puede plantear un programa de intervención común (Aguirre, 1990).

Existen dos tipos de métodos que pueden ser usados para construir una tipología: (i) el "método positivista" (Mignolet et al., 2001) basado en el análisis estadístico de las encuestas a fincas y el "método constructivista" donde los tipos son construidos a partir del conocimiento especializado y luego es validado por las encuestas.

En el año 2008 se propone en una investigación usar el "método positivista" para construir una clasificación de fincas basada en la información estructural y los métodos estadísticos. En la práctica, esta clasificación necesita ser validada por los participantes involucrados, por ejemplo los consultores agrícolas o los miembros de instituciones agrícolas. Luego, una encuesta es aplicada a una muestra de fincas en cada clase de unidad tipológica de producción, habilitada a ser construida usando el "método constructivista". La unidad tipológica de producción es luego validada por la encuesta a la finca y por los participantes. Subsecuentemente, la tipología finca puede ser construida usando el "método positivista" por la combinación de unidades de producción en una muestra finca. La tipología finca es luego extendida a toda la población usando la clasificación de finca.

Por otro lado, Reed et al. (2009) plantean que para ayudar a racionalizar las bases teóricas para el análisis de los agricultores, la metodología instrumental y normativa debe ser aplicada en diferentes disciplinas y contextos usando una amplia variedad de métodos que pueden ser

\footnotetext{
${ }^{1}$ Facultad de Agronomía. Universidad Nacional Agraria La Molina, Lima, Perú. Email: cecilia_figueroa@lamolina.edu.pe

${ }^{2}$ Facultad de Economía y Planificación. Universidad Nacional Agraria La Molina, Lima, Perú. Email: nvaras@lamolina.edu.pe
} 
categorizados como métodos para: (i) la identificación de agricultores; (ii) la diferenciación y categorización entre agricultores y (iii) la investigación relacionada entre agricultores proveyendo una descripción resumida de cada uno de los métodos cubiertos en la tipología, incluyendo detalles de las fuentes requeridas, el nivel de participación de los agricultores y sus fortalezas y debilidades.

Asimismo, Valbuena et al. (2008) señalan que las tipologías se construyen siguiendo los siguientes pasos: (i) los objetivos de modelización del agente: lo cual sirve para entender las diferentes estrategias que los agricultores han seguido y/o deben seguir en términos de la diversificación agrícola practicada en la expansión de la finca; (ii) los criterios de selección: tanto las variables socio económicas como las actitudinales son usadas como criterios para definir los diferentes tipos de agente; (iii) definición del tipo de agente: basada en un análisis de la diversidad de visiones y condiciones socioeconómicas, un "árbol de clasificación" y (iv) estandarización del tipo de agente: después de definir los tipos de agente, estos se estandarizan basados en variables adicionales tales como edad, educación, área cultivada, tipo de agronegocio, sucesores, decisiones de uso de la tierra, membresía de diferentes organizaciones, conocimiento de diferentes proyectos agrícolas, etc.

En el diagnóstico de un sistema agrario, se establece como necesaria la construcción de tipologías para identificar los objetivos de los tipos de agricultores y los mecanismos de toma de decisiones ante lo técnico, para plantear un programa de intervención de mediano plazo adecuado a cada uno con contenidos diferenciados, referentes técnicos apropiados y líneas de investigación agronómica y socioeconómica (Aguirre, 1990).

ElSubproyecto 5 sobre CambioClimático, correspondiente al Proyecto 1 en Investigación de Sistemas Agrarios del Programa UIC/VLIR - UNALM, planteó en el 2010 un estudio sobre el impacto del calentamiento global en la biodiversidad de San Andrés de Tupicocha para conocer cómo las variaciones climáticas influirían sobre las zonas homogéneas de producción, es decir, la altitud en la que los cultivos podrían alcanzar buenos rendimientos. Esta información permitiría a los beneficiarios plantear mecanismos de mitigación al cambio climático por medio de estrategias de manejo de los cultivos. Dicho estudio se realizó por etapas, siendo la primera la identificación y sondeo rural rápido y la segunda la clasificación de los productores.

Por esta razón, el objetivo de este trabajo ha sido desarrollar una tipología de los agricultores de las fincas para categorizar la diversidad de estrategias de vida entre los minifundistas del distrito de San Andrés de Tupicocha. Para este fin, analizamos la tipología en términos de su capacidad para distinguir patrones en el manejo de los recursos como los cultivos y el agua. También se evaluó el aspecto económico y el estudio de la variabilidad en el manejo del agua de riego dentro de cada grupo.

\section{Materiales y métodos}

\section{Área de estudio}

El distrito de San Andrés de Tupicocha se encuentra ubicado entre los 3200 y $4400 \mathrm{msnm}$ y su espacio se encuentra distribuido en tres pisos agroecológicos (Tabla 1): parte alta (zona de pastizales), media (zona de tubérculos) y baja (zona de frutales) en donde se puede observar la diversidad de recursos naturales (UNALM/ VLIR, 2010).

El estudio se desarrolló en la Comunidad de San Andrés de Tupicocha, en el distrito del mismo nombre, ubicado en la parte alta de la cuenca del río Lurín, provincia de Huarochirí, departamento de Lima (Perú); está constituida por 10 parcialidades o ayllus y 8 pueblos anexos (Varas, 2000). Según Gonzáles Holguín (1608) ayllu: “... significa en contextos diferentes: genealogía, linaje, grupo de parentesco, nación, género, especie o clase". Ayllu es diferente a comunidad, esta es una institución rural sin embargo, muchas veces en Perú, Bolivia y Ecuador tratamos ambos términos como sinónimos. San Andrés de Tupicocha alberga un total de 1.423 habitantes, de los cuales 713 son hombres y 710 mujeres (INEI, 2007).

La comunidad está constituida por aproximadamente 400 familias (Tabla 2) (Solomon, 2006), cuya actividad económica prioritaria es la agricultura (81,3\%). Según el INEI (2007) la población se distribuye en un $49,5 \%$ en la zona urbana y un $50,5 \%$ en la zona rural.

\section{Recolección de información y diseño de la encuesta}

Tittonell et al. (2010) recomiendan utilizar variables para la tipología de productores que son incluidas en las encuestas para luego procesarlas estadísticamente; estas son: área total de la propiedad familiar (ha), área total de la finca (ha), área total cultivada (ha), tamaño de la familia (número de miembros que viven y comen en la familia), mano de obra familiar (número de miembros que trabajan en la finca), miembros familiares que trabajan temporal/ permanentemente fuera de la finca, edad de la cabeza de familia, porcentaje del ingreso familiar de actividades no agrícolas, número de años percibiendo ingresos no agrícolas, destino de la producción ( $\%$ de la producción para el mercado), número total de ganado, número de ganado criollo, número de ganado mejorado, número de bueyes y arado de bueyes y meses de autosuficiencia alimentaria.

Sin embargo, las encuestas diseñadas recogieron algunos aspectos socio-económicos (venta de mano de obra, acceso a la educación secundaria, recursos financieros y desarrollo de actividades no agropecuarias) y de manejo de recursos de cada finca por carecer de financiamiento y tiempo. Un grupo de estudiantes de Agronomía del curso de Extensión y Promoción Agraria de la Universidad Nacional Agraria La Molina-UNALM, fue entrenado previamente para aplicarlas. La información sólo incluyó criterios tales como la extensión de la propiedad familiar, el manejo de la biodiversidad, la utilización de insumos agropecuarios, la disponibilidad de agua de riego, el 
Tabla 1. Distribución de los recursos naturales por piso agroecológico

\begin{tabular}{|c|c|c|c|}
\hline & Parte alta & Parte media & Parte baja \\
\hline $\begin{array}{l}\text { Distrito, } \\
\text { comunidades, anexos, } \\
\text { etc. }\end{array}$ & $\begin{array}{l}\text { San Miguel (cerro) y el } \\
\text { caserío Casama }\end{array}$ & $\begin{array}{l}\text { Caserío Casama, caserío } \\
\text { Ansugay, distrito Tupicocha }\end{array}$ & $\begin{array}{l}\text { Distrito Tupicocha y z } \\
\text { Macazán }\end{array}$ \\
\hline Suelos & Tierra negra y fina & Arcilloso, arenoso y fértil & Arcilloso, arenoso y fé \\
\hline Agua & $\begin{array}{l}\text { Riachuelos, manantiales, } \\
\text { etc. Siembra de agua, } \\
\text { lluvias de enero a marzo }\end{array}$ & Riachuelos, manantiales, etc. & $\begin{array}{l}\text { Riachuelos, manantia } \\
\text { etc. }\end{array}$ \\
\hline \multirow[b]{2}{*}{ Animales } & $\begin{array}{l}\text { Taya, chisque, conuco, } \\
\text { taurish, ichu, pastos } \\
\text { naturales, trébol, tucusmin, } \\
\text { queñual, quishuar, sauco. }\end{array}$ & $\begin{array}{l}\text { Papa, oca, olluco, ajo, haba, } \\
\text { cereales, alfalfa, flores, } \\
\text { hierbas aromáticas, eucalipto, } \\
\text { pino, sauco, ciprés, aliso. }\end{array}$ & $\begin{array}{l}\text { Manzano, melocoton } \\
\text { ciruelo, tuna, membrill } \\
\text { palto, chirimoyo, mo } \\
\text { mito, tara, pitajaya, lloc } \\
\text { sauce, etc. }\end{array}$ \\
\hline & $\begin{array}{l}\text { Vicuña, vizcacha, zorro, } \\
\text { cóndor. }\end{array}$ & $\begin{array}{l}\text { Vaca, oveja, cabra, burro, cuy, } \\
\text { conejo, aves de corral, etc. }\end{array}$ & Vaca, cabra \\
\hline \multicolumn{4}{|c|}{$\begin{array}{l}\text { Fuente: UNALM/VLIR. Informe del Taller Rural Participativo de Diagnóstico 2010. Proyecto: Investigación en Sistemas Agrarios. Subproye } \\
\text { Cambio Climático. }\end{array}$} \\
\hline \multicolumn{4}{|c|}{ Tabla 2. Número de jefes de familia de la Comunidad Campesina San Andrés de Tupicocha } \\
\hline \multicolumn{2}{|c|}{ Parcialidades que la integran Miembros activos } & s Miembros pasivos o jubilados & Miembros total \\
\hline 1.Primera Allauca & 9 & 12 & 21 \\
\hline 2. Primera Stafasca & 17 & 9 & 26 \\
\hline 3. Primer Huangre & 6 & 8 & 14 \\
\hline 4. Unión Chauca colca & 27 & 30 & 57 \\
\hline 5. Mújica & 11 & 14 & 25 \\
\hline 6. Cacarima & 27 & 15 & 42 \\
\hline 7. Segunda Allauca & 14 & 12 & 26 \\
\hline 8. Segunda Satafasca & 13 & 6 & 19 \\
\hline 9. Centro Huangre & 28 & 27 & 55 \\
\hline 10. Huangre Boys & 12 & 11 & 23 \\
\hline Total & 164 & 144 & 308 \\
\hline
\end{tabular}

destino de la producción agrícola y pecuaria, la venta de la mano de obra, el acceso a la educación secundaria, los recursos financieros: préstamos, cuentas de ahorros, etc. y el desarrollo de actividades no agropecuarias: panadería, artesanía, etc.

La recolección de la información se realizó durante el mes de agosto del 2010 recogiéndose 50 encuestas aplicadas a productores jefes de familia, esto significó una muestra del $13 \%$.

\section{Análisis estadístico}

Las referencias señalan como método el análisis de componente principal (PCA) para identificar indicadores socioeconómicos no correlacionados a ser usados luego como lo más representativo de los criterios de categorización de las familias y agruparlas en clases homogéneas, usando repetidamente el análisis de conglomerado no jerárquico (CA) complementándolo con la reclasificación "a mano" de casos poco claros (Gaspar et al., 2008; Van Herzele \& Van Gossum, 2008 y
Tittonell et al., 2010). Sin embargo, en el presente estudio descriptivo hemos trabajado solamente con el análisis de frecuencias porque las variables evaluadas han sido solamente cualitativas y la clasificación de agricultores ha sido hecha a mano utilizando los porcentajes más destacados.

La Tabla de Frecuencias fue usada para organizar la información en clases, es decir en grupos de valores que describen una característica de los datos y muestran el número de observaciones del conjunto de datos que cae en cada una de las clases. Asimismo, ayuda a agrupar cualquier tipo de dato numérico. En principio, en esta se detalla cada uno de los valores diferentes en el conjunto de datos junto con el número de veces que aparece, es decir, su frecuencia absoluta. Se puede complementar la frecuencia absoluta con la denominada frecuencia relativa, que indica la frecuencia en porcentaje sobre el total de datos.

El paquete estadístico SPSS (versión 15.0) fue utilizado para el análisis. El análisis de frecuencias fue aplicado a 45 variables cualitativas. 


\section{Clasificación de agricultores}

Generalmente, en la construcción de tipologías se utilizan encuestas que incluyen variables cualitativas y sobretodo cuantitativas que permitan realizar las estimaciones necesarias para definir las diferencias y las similitudes entre los tipos de agricultores.

En el caso de nuestra investigación, se plantearon 9 indicadores distribuidos en 45 variables cualitativas para establecer mediante el análisis de frecuencias aquellas que destacaban en porcentaje y con dicha información poder agrupar a los agricultores. Estos son: extensión de terreno, diversidad de cultivos, utilización de insumos agropecuarios en los procesos de producción, disponibilidad de agua de riego, destino de la producción, mano de obra, grado de instrucción, disponibilidad de recursos financieros y desarrollo de actividades no agropecuarias complementarias.

Se elaboraron matrices según las variables categorizadas y luego se agruparon por las similitudes encontradas obteniéndose clases.

\section{Resultados y discusión}

Análisis de variables cualitativas

Diversidad de cultivos. En la Tabla 3 se puede observar que el grupo de agricultores que poseen entre 3,0 a 9,9

Tabla 3. Diversidad de cultivos por rango de área de los agricultores de San Andrés de Tupicocha

\begin{tabular}{|c|c|c|c|c|c|}
\hline & $\begin{array}{c}\text { Menos de } \\
0,5 \text { ha }\end{array}$ & $\begin{array}{c}\text { De } 0,5 \text { a } \\
0,9 \text { ha }\end{array}$ & $\begin{array}{c}\text { De } 1,0 \text { a } \\
1,9 \text { ha }\end{array}$ & $\begin{array}{c}\text { De } 2,0 \text { a } \\
2,9 \mathrm{ha}\end{array}$ & $\begin{array}{c}\text { De } 3,0 \text { a } \\
9,9 \text { ha }\end{array}$ \\
\hline \multicolumn{6}{|l|}{ Papa } \\
\hline Frecuencia & 8 & 7 & 7 & 10 & 3 \\
\hline Total & 14 & 11 & 11 & 11 & 3 \\
\hline Porcentaje & 57,1 & 63,6 & 63,6 & 90,9 & 100,0 \\
\hline \multicolumn{6}{|l|}{ Mashua } \\
\hline Frecuencia & 2 & 3 & 3 & 1 & 3 \\
\hline Total & 14 & 11 & 11 & 11 & 3 \\
\hline Porcentaje & 14,3 & 27,3 & 27,3 & 9,1 & 100,0 \\
\hline \multicolumn{6}{|l|}{ Oca } \\
\hline Frecuencia & 5 & 4 & 6 & 9 & 2 \\
\hline Total & 14 & 11 & 11 & 11 & 3 \\
\hline Porcentaje & 35,7 & 36,4 & 54,5 & 81,8 & 66,7 \\
\hline \multicolumn{6}{|l|}{ Olluco } \\
\hline Frecuencia & 3 & 3 & 2 & 4 & 1 \\
\hline Total & 14 & 11 & 11 & 11 & 3 \\
\hline Porcentaje & 21,4 & 27,3 & 18,2 & 36,4 & 33,3 \\
\hline \multicolumn{6}{|l|}{ Cereal } \\
\hline Frecuencia & 4 & 2 & 5 & 4 & 2 \\
\hline Total & 14 & 11 & 11 & 11 & 3 \\
\hline Porcentaje & 28,6 & 18,2 & 45,5 & 36,4 & 66,7 \\
\hline \multicolumn{6}{|l|}{ Haba } \\
\hline Frecuencia & 5 & 6 & 4 & 6 & 3 \\
\hline Total & 14 & 11 & 11 & 11 & 3 \\
\hline Porcentaje & 35,7 & 54,5 & 36,4 & 54,5 & 100,0 \\
\hline \multicolumn{6}{|l|}{ Arveja } \\
\hline Frecuencia & 2 & 1 & 3 & 3 & 2 \\
\hline Total & 14 & 11 & 11 & 11 & 3 \\
\hline Porcentaje & 14,3 & 9,1 & 27,3 & 27,3 & 66,7 \\
\hline \multicolumn{6}{|l|}{ Maíz } \\
\hline Frecuencia & 4 & 2 & 5 & 4 & 3 \\
\hline Total & 14 & 11 & 11 & 11 & 3 \\
\hline Porcentaje & 28,6 & 18,2 & 45,5 & 36,4 & 100,0 \\
\hline \multicolumn{6}{|l|}{ Frutales } \\
\hline Frecuencia & 10 & 9 & 11 & 9 & 2 \\
\hline Total & 14 & 11 & 11 & 11 & 3 \\
\hline Porcentaje & 71,4 & 81,8 & 100,0 & 81,8 & 66,7 \\
\hline \multicolumn{6}{|l|}{ Otros cultivos } \\
\hline Frecuencia & 3 & 3 & 3 & 8 & 2 \\
\hline Total & 14 & 11 & 11 & 11 & 3 \\
\hline Porcentaje & 21,4 & 27,3 & 27,3 & 72,7 & 66,7 \\
\hline
\end{tabular}


ha son los que manejan porcentajes mayores de papa, oca, olluco, cereales, haba, arveja, maíz, frutales y otros; mientras que el grupo de agricultores que tienen menos de 0,5 ha manejan un menor porcentaje de cultivos. El incremento de los porcentajes de los cultivos se va haciendo paulatino a medida que aumenta la extensión de los predios. Como podemos observar, existe una relación directa entre la extensión del área y el porcentaje de la diversidad de cultivos.

Utilización de insumos agropecuarios. En la Tabla 4 se observa que el fertilizante orgánico es un insumo que es utilizado en más del $80 \%$ por todos los agricultores encuestados. Por otro lado, aquellos que se encuentran en el rango de 1,0 a 1,9 ha utilizan la mayor cantidad de insumos agropecuarios tales como: semillas, pesticidas, fertilizantes, entre otros. En cambio, los agricultores que están en el rango de 3,0 a 9,9 ha sólo utilizan semilla, insecticida y fertilizante orgánico.
Disponibilidad de agua de riego. En el área de estudio, existe un sistema muy complejo de manejo y gestión del agua, la cual proviene de distintas fuentes: lluvia, deshielo, amunas, reservorio, otros. Las amunas podemos tipificarlas como sistemas de recarga superficial localizados fuera de los cauces de los ríos, mediante sistemas de canales asociados a campos de extensión, cuyo principio es captar las aguas del río, llevarlo fuera del cauce mediante canales y extenderlos en una superficie permeable que puede ser un desierto, áreas de bajo valor agrícola, zonas pedregosas de gran extensión, que se asemejan a la metodología de las amunas practicadas por nuestros antepasados. Estas se habrían constituido en eficaces sistemas de recarga artificial utilizados en los Andes peruanos, en lugares donde la escasez de agua era aguda, y se disponía de rocas acuíferas que permitían su almacenamiento subterráneo, para usarlas aguas abajo en las épocas de mayor demanda y ausencia de lluvias (Apaza et al., 2006).

Tabla 4. Utilización de insumos agropecuarios por rango de área de los agricultores de San Andrés de Tupicocha

\begin{tabular}{|c|c|c|c|c|c|}
\hline & Menos de 0,5ha & De 0,5 a $0,9 \mathrm{ha}$ & De 10 a $1,9 \mathrm{ha}$ & De 2,0 a $2,9 \mathrm{ha}$ & De 3,0 a 9,9ha \\
\hline \multicolumn{6}{|l|}{ Semilla } \\
\hline Frecuencia & 7 & 6 & 8 & 10 & 2 \\
\hline Total & 14 & 11 & 11 & 11 & 3 \\
\hline Porcentaje & 50,0 & 54,5 & 72,7 & 90,9 & 66,7 \\
\hline \multicolumn{6}{|l|}{ Insecticida } \\
\hline Frecuencia & 3 & 4 & 6 & 3 & 2 \\
\hline Total & 14 & 11 & 11 & 11 & 3 \\
\hline Porcentaje & 21,4 & 36,4 & 54,5 & 27,3 & 66,7 \\
\hline \multicolumn{6}{|l|}{ Herbicida } \\
\hline Frecuencia & 0 & 4 & 3 & 2 & 0 \\
\hline Total & 14 & 11 & 11 & 11 & 14 \\
\hline Porcentaje & 0,0 & 36,4 & 27,3 & 18,2 & 0,0 \\
\hline \multicolumn{6}{|l|}{ Fungicida } \\
\hline Frecuencia & 1 & 1 & 3 & 0 & 0 \\
\hline Total & 14 & 11 & 11 & 11 & 3 \\
\hline Porcentaje & 7,1 & 9,1 & 27,3 & 0,0 & 0,0 \\
\hline \multicolumn{6}{|l|}{ Fertilizantes minerales } \\
\hline Frecuencia & 1 & 3 & 5 & 5 & 0 \\
\hline Total & 14 & 11 & 11 & 11 & 3 \\
\hline Porcentaje & 7,1 & 27,3 & 45,5 & 45,5 & 0,0 \\
\hline \multicolumn{6}{|l|}{ Fertilizantes orgánicos } \\
\hline Frecuencia & 13 & 10 & 10 & 11 & 3 \\
\hline Total & 14 & 11 & 11 & 11 & 3 \\
\hline Porcentaje & 92,9 & 90,9 & 90,9 & 100,0 & 100,0 \\
\hline \multicolumn{6}{|l|}{ Defoliante } \\
\hline Frecuencia & 2 & 0 & 1 & 1 & 0 \\
\hline Total & 14 & 11 & 11 & 11 & 3 \\
\hline Porcentaje & 14,3 & 0,0 & 9,1 & 9,1 & 0,0 \\
\hline \multicolumn{6}{|l|}{ Otros insumos } \\
\hline Frecuencia & 0 & 0 & 0 & 2 & 0 \\
\hline Total & 14 & 11 & 11 & 11 & 3 \\
\hline Porcentaje & 0,0 & 0,0 & 0,0 & 18,2 & 0,0 \\
\hline
\end{tabular}


Los agricultores son miembros de la Comisión de Regantes, lo que les da derecho a acceder a agua de los canales de riego. Sin embargo, entre ellos hay diferencias que se ven reflejadas en los datos obtenidos. Todos los agricultores encuestados utilizan agua de riego (Tabla 5), pero son los del rango entre 3,0 a 9,9 ha los que usan un mayor porcentaje de distintos tipos de fuentes, es decir agua proveniente de canales, amunas, reservorios y otros.

Destino de la producción agrícola y pecuaria. En cuanto al destino de la producción agrícola y pecuaria tenemos, en la Tabla 6, a los pequeños productores que destinan los productos agropecuarios tanto al autoconsumo como a la venta a los mercados de Lima. Sin embargo, encontramos que los agricultores que están dentro del rango de 3,0 a 9,9 ha, son los que destinan en un $100 \%$ la producción de alfalfa para la venta fuera del distrito, no sin dejar de utilizar otros cultivos para su autoconsumo (papa, haba, trigo, oca y arveja). En cambio, los demás grupos consumen más sus productos agrícolas (más de $78 \%$ de agricultores) y en menor medida los productos pecuarios (apenas el 18 $\%$ de agricultores en el rango de 1,0 a 1,9 ha) en lugar de venderlos fuera de Tupicocha. Este comportamiento, quizás, se deba a la escasa infraestructura de carreteras que impide la libre circulación de los productos.
Mano de obra. En la zona existe un mercado de trabajo, todos los agricultores venden su mano de obra para obtener recursos monetarios que complementen sus ingresos familiares. De ahí que todos los agricultores vendan su mano de obra en la actividad agrícola entre un 30 y un $50 \%$; sin embargo los que poseen extensiones entre 1,0 a 2,9 ha venden su mano de obra tanto en la actividad agrícola como pecuaria (Tabla 7).

Grado de instrucción. En el distrito existe una institución de educación secundaria estatal a la que tienen acceso los hijos de los comuneros. Pero hemos encontrado que los agricultores que se encuentran entre las 3,0 y las 9,9 ha manifestaron no tener acceso a los estudios secundarios, probablemente se deba a que cuentan con recursos económicos para el sostenimiento de sus familias, prescindiendo del grado de instrucción, o se deba a que cuando eran jóvenes no existía secundaria en Tupicocha. Lo que no sucede con los agricultores encuestados que poseen menor extensión. Los agricultores que se encuentran en el rango de 1,0 a 1,9 ha señalaron tener educación secundaria completa en mayor porcentaje a todos los demás grupos (Tabla 8).

Recursos financieros. En la Tabla 9 se observa que el hábito del ahorro y del préstamo no es una característica

Tabla 5. Disponibilidad de agua de riego entre los agricultores de San Andrés de Tupicocha

\begin{tabular}{|c|c|c|c|c|c|}
\hline & $\begin{array}{c}\text { Menos de } \\
\mathbf{0 , 5 h a}\end{array}$ & $\begin{array}{c}\text { De } 0,5 \text { a } \\
0,9 \text { ha }\end{array}$ & $\begin{array}{c}\text { De } 1,0 \text { a } \\
1,9 \text { ha }\end{array}$ & $\begin{array}{l}\text { De } 2,0 \text { a } \\
2,9 \text { ha }\end{array}$ & $\begin{array}{c}\text { De 3,0 a } \\
9,9 \text { ha }\end{array}$ \\
\hline $\begin{array}{l}\text { Agua de riego } \\
\text { Frecuencia }\end{array}$ & 12 & 10 & 11 & 11 & 3 \\
\hline Total & 14 & 11 & 11 & 11 & 3 \\
\hline Porcentaje & 85,7 & 90,9 & 100,0 & 100,0 & 100,0 \\
\hline \multicolumn{6}{|l|}{ Canal } \\
\hline Frecuencia & 3 & 2 & 4 & 4 & 2 \\
\hline Total & 14 & 11 & 11 & 11 & 3 \\
\hline Porcentaje & 21,4 & 18,2 & 36,4 & 36,4 & 66,7 \\
\hline \multicolumn{6}{|l|}{ Amuna } \\
\hline Frecuencia & 4 & 8 & 9 & 3 & 2 \\
\hline Total & 14 & 11 & 11 & 11 & 3 \\
\hline Porcentaje & 28,6 & 72,7 & 81,8 & 27,3 & 66,7 \\
\hline \multicolumn{6}{|l|}{ Reservorio } \\
\hline Frecuencia & 5 & 3 & 5 & 8 & 1 \\
\hline Total & 14 & 11 & 11 & 11 & 3 \\
\hline Porcentaje & 35,7 & 27,3 & 45,5 & 72,7 & 33,3 \\
\hline \multicolumn{6}{|l|}{ Otro } \\
\hline Frecuencia & 2 & 0 & 2 & 0 & 3 \\
\hline Total & 14 & 11 & 11 & 11 & 3 \\
\hline Porcentaje & 14,3 & 0,0 & 18,2 & 0,0 & 100,0 \\
\hline
\end{tabular}


Tabla 6. Destino de la producción agrícola y pecuaria de los agricultores de San Andrés de Tupicocha

\begin{tabular}{|c|c|c|c|c|c|}
\hline & $\begin{array}{c}\text { Menos de } \\
\mathbf{0 , 5 h a}\end{array}$ & $\begin{array}{c}\text { De } 0,5 \text { a } \\
0,9 \text { ha }\end{array}$ & $\begin{array}{c}\text { De } 1,0 \text { a } \\
1,9 h a\end{array}$ & $\begin{array}{c}\text { De } 2,0 \text { a } \\
2,9 \mathrm{ha}\end{array}$ & $\begin{array}{c}\text { De } 3,0 \text { a } \\
9,9 \text { ha }\end{array}$ \\
\hline \multicolumn{6}{|l|}{ Autoconsumo agrícola } \\
\hline Frecuencia & 11 & 9 & 9 & 9 & 3 \\
\hline Total & 14 & 11 & 11 & 11 & 3 \\
\hline Porcentaje & 78,6 & 81,8 & 81,8 & 81,8 & 100,0 \\
\hline \multicolumn{6}{|l|}{ Autoconsumo pecuario } \\
\hline Frecuencia & 0 & 0 & 2 & 0 & 0 \\
\hline Total & 14 & 11 & 11 & 11 & 3 \\
\hline Porcentaje & 0,0 & 0,0 & 18,2 & 0,0 & 0,0 \\
\hline \multicolumn{6}{|l|}{ Mercado local agrícola } \\
\hline Frecuencia & 3 & 3 & 4 & 2 & 0 \\
\hline Total & 14 & 11 & 11 & 11 & 3 \\
\hline Porcentaje & 21,4 & 27,3 & 36,4 & 18,2 & 0,0 \\
\hline \multicolumn{6}{|l|}{ Mercado local pecuario } \\
\hline Frecuencia & 0 & 0 & 0 & 1 & 0 \\
\hline Total & 14 & 11 & 11 & 11 & 3 \\
\hline Porcentaje & 0,0 & 0,0 & 0,0 & 9,1 & 0,0 \\
\hline \multicolumn{6}{|l|}{ Mercado externo agrícola } \\
\hline Frecuencia & 7 & 5 & 8 & 5 & 3 \\
\hline Total & 14 & 11 & 11 & 11 & 3 \\
\hline Porcentaje & 50,0 & 45,5 & 72,7 & 45,5 & 100,0 \\
\hline \multicolumn{6}{|l|}{ Mercado externo pecuario } \\
\hline Frecuencia & 1 & 0 & 2 & 1 & 0 \\
\hline Total & 14 & 11 & 11 & 11 & 3 \\
\hline Porcentaje & 7,1 & 0,0 & 18,2 & 9,1 & 0,0 \\
\hline
\end{tabular}

visible entre los agricultores que poseen grandes extensiones de terreno. Asimismo, los agricultores que están en el rango de 0,5 a 1,9 ha llegan a prestarse dinero de aquellos que sí lo tienen, posiblemente para cubrir los gastos de la campaña agrícola.

Desarrollo de actividades no agropecuarias. En Tupicocha, los agricultores no se dedican exclusivamente a las actividades agropecuarias. Todos los que respondieron a las encuestas declararon tener actividades complementarias a ellas como son poseer pequeños negocios en el área urbana.

Así, los agricultores que están entre las 3,0 y las 9,9 ha señalaron tener bodegas y restaurantes, como actividades no agropecuarias, que les genera ingresos y liquidez (Tabla 10). Otras actividades no agropecuarias que les generan ingresos son: talleres de corte y confección, docencia escolar, entre otros; sobre todo a los grupos que tienen menores extensiones de terreno.

\section{Clasificación de agricultores}

Clase 1

Los agricultores que pertenecen a esta clase poseen menos de 0,5 ha de terreno y siembran en ella principalmente papa y frutales. Utilizan en cada una de sus campañas agrícolas una serie de insumos agrícolas, pero destacan la semilla y el fertilizante orgánico. Usan agua de riego proveniente del reservorio, como fuente principal. Destinan su producción agrícola al autoconsumo y al mercado externo (i.e. Lima). Ofertan su mano de obra para las labores agrícolas en un $50 \%$. Los agricultores de esta clase han cursado la secundaria; algunos (40\%) no la han terminado, mientras que otros (40 \%) sí. Dentro de sus recursos financieros, los agricultores que ahorran son aproximadamente un $50 \%$. Entre las actividades no agropecuarias que desarrollan está la administración de bodegas $(20 \%)$, entre otras actividades $(20 \%)$. 
Tabla 7. Oferta de la mano de obra

\begin{tabular}{|c|c|c|c|c|c|}
\hline & $\begin{array}{c}\text { Menos de } \\
\mathbf{0 , 5 h a}\end{array}$ & $\begin{array}{c}\text { De } 0,5 \text { a } \\
0,9 \text { ha }\end{array}$ & $\begin{array}{c}\text { De } 1,0 \text { a } \\
1,9 h a\end{array}$ & $\begin{array}{c}\text { De } 2,0 \text { a } \\
2,9 \mathrm{ha}\end{array}$ & $\begin{array}{c}\text { De } 3,0 \text { a } \\
9,9 \mathrm{ha}\end{array}$ \\
\hline \multicolumn{6}{|l|}{ Trabajo agrícola } \\
\hline Frecuencia & 7 & 3 & 3 & 6 & 1 \\
\hline Total & 14 & 11 & 11 & 11 & 3 \\
\hline Porcentaje & 50,0 & 27,3 & 27,3 & 54,5 & 33,3 \\
\hline \multicolumn{6}{|l|}{ Trabajo pecuario } \\
\hline Frecuencia & 0 & 0 & 1 & 1 & 0 \\
\hline Total & 14 & 11 & 11 & 11 & 3 \\
\hline Porcentaje & 0,0 & 0,0 & 9,1 & 9,1 & 0,0 \\
\hline
\end{tabular}

Tabla 8. Grado de instrucción

\begin{tabular}{rrrrrr}
\hline & $\begin{array}{c}\text { Menos de } \\
\text { 0,5ha }\end{array}$ & $\begin{array}{c}\text { De 0.5 a } \\
\text { 0,9ha }\end{array}$ & $\begin{array}{c}\text { De 1.0 a } \\
\mathbf{1 , 9 h a}\end{array}$ & $\begin{array}{c}\text { De 2.0 a } \\
\mathbf{2 , 9 h a}\end{array}$ & $\begin{array}{c}\text { De 3.0 a } \\
\mathbf{9 , 9 h a}\end{array}$ \\
\hline Sin instrucción & & & & & \\
Frecuencia & 2 & 3 & 3 & 3 & 3 \\
Total & 14 & 11 & 11 & 11 & 3 \\
Porcentaje & 14,3 & 27,3 & 27,3 & 27,3 & 100,0
\end{tabular}

Secundaria incompleta

$\begin{array}{rrrrrr}\text { Frecuencia } & 6 & 4 & 1 & 5 & 0 \\ \text { Total } & 14 & 11 & 11 & 11 & 3 \\ \text { Porcentaje } & 42,9 & 36,4 & 9,1 & 45,5 & 0,0\end{array}$

Secundaria completa

\begin{tabular}{rrrrrr} 
Frecuencia & 6 & 4 & 7 & 3 & 0 \\
Total & 14 & 11 & 11 & 11 & 3 \\
Porcentaje & 42,9 & 36,4 & 63,6 & 27,3 & 0,0 \\
\hline
\end{tabular}

Tabla 9. Recursos financieros de los agricultores de San Andrés de Tupicocha

\begin{tabular}{ccccc}
\hline Menos de & De 0,5 a & De 1,0 a & De 2,0 a & De 3,0 a \\
0,5ha & 0,9 ha & 1,9 ha & 2,9 ha & 9,9 ha \\
\hline
\end{tabular}

\section{Ahorro}

$\begin{array}{rrrrrr}\text { Frecuencia } & 7 & 8 & 8 & 5 & 0 \\ \text { Total } & 14 & 11 & 11 & 11 & 3 \\ \text { Porcentaje } & 50,0 & 72,7 & 72,7 & 45,5 & 0,0\end{array}$

\section{Préstamo}

$\begin{array}{rrrrrr}\text { Frecuencia } & 0 & 1 & 1 & 0 & 0 \\ \text { Total } & 14 & 11 & 11 & 11 & 3 \\ \text { Porcentaje } & 0,0 & 9,1 & 9,1 & 0,0 & 0,0\end{array}$


Tabla 10. Actividades no agropecuarias entre los agricultores de San Andrés de Tupicocha

\begin{tabular}{|c|c|c|c|c|c|}
\hline & $\begin{array}{c}\text { Menos de } \\
0,5 \text { ha }\end{array}$ & $\begin{array}{c}\text { De 0,5 a } \\
0,9 \text { ha }\end{array}$ & $\begin{array}{c}\text { De } 1,0 \text { a } \\
1,9 \text { ha }\end{array}$ & $\begin{array}{c}\text { De 2,0 a } \\
2,9 \text { ha }\end{array}$ & $\begin{array}{c}\text { De 3,0 a } \\
9,9 \text { ha }\end{array}$ \\
\hline \multicolumn{6}{|l|}{ Bodega } \\
\hline Frecuencia & 3 & 2 & 1 & 2 & 1 \\
\hline Total & 14 & 11 & 11 & 11 & 3 \\
\hline Porcentaje & 21,4 & 18,2 & 9,1 & 18,2 & 33,3 \\
\hline \multicolumn{6}{|l|}{ Restaurante } \\
\hline Frecuencia & 0 & 0 & 0 & 0 & 1 \\
\hline Total & 14 & 11 & 11 & 11 & 3 \\
\hline Porcentaje & 0,0 & 0,0 & 0,0 & 0,0 & 33,3 \\
\hline \multicolumn{6}{|l|}{ Transporte } \\
\hline Frecuencia & 0 & 1 & 0 & 0 & 0 \\
\hline Total & 14 & 11 & 11 & 11 & 3 \\
\hline Porcentaje & 0,0 & 9,1 & 0,0 & 0,0 & 0,0 \\
\hline \multicolumn{6}{|l|}{ Otra actividad } \\
\hline Frecuencia & 3 & 1 & 1 & 1 & 0 \\
\hline Total & 14 & 11 & 11 & 11 & 3 \\
\hline Porcentaje & 21,4 & 9,1 & 9,1 & 9,1 & 0,0 \\
\hline
\end{tabular}

\section{Clase 2}

Los agricultores de esta clase tienen una extensión de terreno entre 0,5 a 0,9 ha y siembran en ella toda clase de cultivos (papa, mashua, oca, olluco, cereales, haba, maíz, frutales, etc.) pero destacan la papa, el haba y los frutales. Entre los insumos agropecuarios que más utilizan están la semilla y el fertilizante orgánico. Los agricultores utilizan agua de riego proveniente de las amunas. Los productos agrícolas se destinan al autoconsumo, al mercado local (en el mismo distrito de San Andrés de Tupicocha) y al mercado externo (i.e. Lima). Los agricultores venden su mano de obra al trabajo agrícola, en un $20 \%$. En cuanto al grado o nivel de instrucción, menos del 40\% de los agricultores han cursado la secundaria, pero no la concluyeron, y en la misma proporción la terminaron (menos del $40 \%$ ). El $20 \%$ restante ha señalado no haber cursado la secundaria. Más del $70 \%$ de los agricultores tienen el hábito de ahorrar y se dedican a la administración de las bodegas en menos del $20 \%$ y en un $10 \%$ al transporte y a otra actividad.

\section{Clase 3}

Los agricultores que corresponden a esta clase poseen entre 1,0 a 1,9 ha de terreno. Cultivan toda clase de cultivos, pero destacan la papa, la oca, los cereales, el maíz y los frutales. Entre los insumos agropecuarios más utilizados están la semilla, el insecticida, el fertilizante mineral y el fertilizante orgánico. Los agricultores utilizan el agua de riego de las amunas y de los reservorios. La producción agrícola se destina al autoconsumo, al mercado local y al mercado externo (i.e. Lima). Los agricultores venden su mano de obra en un $25 \%$ a las labores agrícolas y en un 10
$\%$ a las labores pecuarias. El $60 \%$ de los agricultores ha terminado la secundaria. Entre los recursos financieros, el ahorro es practicado por más del $70 \%$ de los agricultores y el préstamo por un $10 \%$ de los mismos. Menos del 10 $\%$ de los agricultores administra una bodega y realiza otra actividad que no es agropecuaria.

\section{Clase 4}

Los agricultores que pertenecen a esta clase se caracterizan por tener entre 2,0 a 2,9 ha. En esta extensión de terreno siembran una diversidad de cultivos, pero destacan la papa, la oca, la haba, los frutales y otros cultivos (i.e. plantas medicinales). Los agricultores de esta clase utilizan como insumos agropecuarios más importantes: la semilla, el fertilizante mineral y el fertilizante orgánico. Los agricultores utilizan agua de riego proveniente de los reservorios. La producción agrícola se destina al autoconsumo y al mercado externo. Casi el $60 \%$ de los agricultores venden su mano de obra en las labores agrícolas y un $10 \%$ a las labores pecuarias. Más del $40 \%$ de los agricultores han cursado la secundaria incompleta. Menos del $50 \%$ de los agricultores ahorran. Más del 15 $\%$ administra una bodega y un $10 \%$ realiza otro tipo de actividad que no es agropecuaria.

\section{Clase 5}

Los agricultores de esta clase poseen entre 3,0 a 9,9 ha de terreno. En dicha extensión siembran toda clase de cultivos entre los que destacan la papa, la oca, los cereales, el haba, la arveja, el maíz, los frutales y otros cultivos. La semilla, el insecticida, el fertilizante orgánico son los insumos agropecuarios más utilizados. 
Los agricultores utilizan agua de riego proveniente de canales, amunas y de otra fuente. La producción agrícola se destina al autoconsumo y al mercado externo (i.e. Lima). El $30 \%$ de los agricultores vende su mano de obra en las labores agrícolas. El $100 \%$ no ha tenido acceso a la educación secundaria. En esta clase, los agricultores carecen de ahorros y préstamos. Sin embargo, más del 30 $\%$ administra una bodega y un restaurante.

Encontramos que entre los indicadores: diversidad de cultivos y disponibilidad de agua de riego, existe una relación directa con la tenencia de la propiedad familiar, ya que a mayor extensión de la propiedad hay mayor disponibilidad de recursos de agua y cultivos. Esto nos haría suponer que los agricultores que pertenecen a las clases 4 y 5 tienen distribuidas sus parcelas en todos los pisos agroecológicos referidos en la Tabla 1.

Por otro lado, la relación entre la utilización de insumos agropecuarios y la venta de mano de obra con la extensión de la propiedad nos indica que los agricultores que poseen menos de 0,5 ha venden su mano de obra en la actividad agrícola en un $50 \%$ y compran semilla en un $50 \%$, insecticida en un $20 \%$, fertilizante mineral y fungicida en un $10 \%$, fertilizante orgánico en un $90 \%$ y defoliante en más del $10 \%$. Hay que agregar que esta clase de agricultores (clase 1) obtienen ingresos de otras actividades como son la administración de bodegas (20 $\%$ ) y en un $20 \%$ a través de la docencia, la carpintería y el servicio doméstico. En cambio, los agricultores de la clase 3 y 4 venden su mano de obra en las actividades agrícolas y pecuarias, contando con la facilidad de adquirir semilla entre un 80 a $90 \%$, insecticida entre un 50 a $20 \%$, herbicida entre un 20 y $10 \%$, fungicida en un $20 \%$, fertilizante mineral en un $40 \%$, fertilizante orgánico entre un 90 a $100 \%$ y defoliante en menos del $10 \%$. Dichos agricultores poseen entre un 9 y $19 \%$ bodegas y realizan otras actividades en casi un $10 \%$. Esto nos demuestra que los agricultores cuentan con diversas estrategias de vida.

En este trabajo de investigación se ha representado la diversidad del sistema agrario de San Andrés de Tupicocha, porque se ha podido identificar cinco grupos de trabajo homogéneos en cuanto a objetivos e intereses.

Por otro lado, para construir la tipología de pequeños agricultores se ha utilizado el método constructivista, citado por Papert (1993), porque primero se ha realizado un Diagnóstico Rural Participativo (DRP) y en base a la información obtenida se han elaborado las encuestas para validar y recoger más datos. Si bien es cierto que Poussin et al. (2008) recomienda utilizar el método positivista, se plantea en la misma cita que en la práctica es necesario validar la información por los pequeños productores para poder extender a todo el distrito la clasificación de finca.

A través de esta clasificación de productores hemos podido identificarlos, diferenciarlos y categorizarlos. Quizás lo que no hemos podido lograr es medir el nivel de participación de los agricultores, sus fortalezas y debilidades. Sin embargo, sí hemos logrado entender a través de la diversificación agrícola y el manejo del agua las diferentes estrategias que los agricultores han practicado. Asimismo, hemos podido construir el "árbol de clasificación" de Valbuena et al. (2008), ya que hemos podido definir el tipo de productor basado en las condiciones socioeconómicas. También se ha logrado estandarizar a los agricultores en función a variables adicionales tales como: diversidad de cultivos, utilización de insumos agropecuarios, disponibilidad de agua de riego, destino de la producción agrícola y pecuaria, mano de obra, grado de instrucción, recursos financieros y desarrollo de actividades no agropecuarias.

\section{Conclusiones}

La tipología de las familias agrícolas esta compuesta por cinco clases de agricultores. Así tenemos a los que manejan más de 3,0 ha de terreno (clase 5) y que tienen el control de la mayor cantidad de la diversidad de cultivos, aunque no utilizan una gran cantidad de insumos y disponen de más de una fuente de agua para el riego de sus cultivos.

Por otro lado, están los agricultores que manejan menos de 0,5 ha y que señalan tener acceso a la educación secundaria, pero que sólo tienen el control de una fuente de agua para el riego de sus cultivos. Poseen más de una estrategia de sostenimiento de sus familias, además de la agricultura, como son la venta de su mano de obra y en el desarrollo de actividades no agropecuarias.

Asimismo, los agricultores que se encuentran en el rango de 1,0 a 2,0 ha manejan una diversidad de cultivos, que es menor en porcentaje que los de la clase 5 y más que los de la clase 1. Disponen de más de una fuente de agua para regar sus cultivos, pero no en la proporción que los de la clase 5 . Sin embargo, están en capacidad de vender su cosecha al mercado local y al externo.

Finalmente, la construcción de tipologías ha permitido obtener información necesaria para identificar a los actores principales que participarán en la modelización de los sistemas agrarios de San Andrés de Tupicocha.

\section{Literatura citada}

Aguirre, A.F. 1990. Tipologías campesinas para la intervención técnica. Agricultura Andina: unidad y sistema de producción. Diálogo entre Ciencias Agrarias y Ciencias Sociales. Lima, Perú: ORSTOM/Editorial Horizonte/UNALM.

Apaza, I.; Arroyo, H. y Alencastre, C. 2006. Las amunas de Huarochirí: recarga de acuíferos en los Andes. Lima: GSAAC/Embajada de Países Bajos/ IICA

Gaspar, P.; Escribano, M.; Mesías, F.J.; Rodríguez de Ledesma, A. y Pulido, F. 2008. Sheep farms in the Spanish rangelands (dehesas): Typologies according to livestock management and economic indicators. Small Ruminant Research, 74: 52 - 63.

González Holguín, D. 1608. Vocabvlario dela lengva general de todo el Perv llamada lengua

Qquichua o del Inca, Lima, Francisco del Canto, 1608.

INEI . 2007. CENSOS. Disponible en: http://censos. inei.gob.pe/cpv2007/tabulados/

Mignolet, C.; Benoît, M. and Bornerand, C. 2001. Différenciation du basin de la Seine selon les dynamiques 
des systèmes de production agricoles depuis les années 70. Agricultures, 10 (6): $377-387$.

Papert, S. 1993. Mindstorms: Children, computers and powerful ideas. Nueva York: Basic Books.

Reed, M.S.; Graves, A.; Dandy, N.; Posthumus, H.; Hubacek, K.; Morris, J.; Prell, C.; Quinn, C. and Stringer, L.C. 2009. Who's in and why? A typology of stakeholder analysis methods for natural resource management. Journal of Environmental Management, 90: $1933-1949$.

Solomon, F. 2006. Los Quipocamayos, el antiguo arte del kipu en una comunidad campesina moderna. Lima: IFEA IEP.

Tittonell, P.; Muriuki, A.; Sheperd, K.D.; Mugendi, D.; Kaizzi, K.C.; Okeyo, J.; Verchot, L.; Coe, R. and Vanlauwe, B. 2010. The diversity of rural livelihoods and their influence on soil fertility in agricultural systems of East Africa - A typology of smallholder farms. Agricultural Systems, 103: 83-97.

UNALM/VLIR. 2010. Informe Taller Rural Participativo de Diagnóstico. Proyecto: Investigación en Sistemas Agrarios. Subproyecto: Cambio Climático.

Valbuena, D.; Verburg, P.H. and Bregt, A. K. 2008. A method to define a typology for agent-based analysis in regional land-use research. Agriculture, Ecosystems and Environment, 128: 27 - 36.

Van Herzele, A. and Van Gossum, P. 2008. Typology building for owner-specific policies and communications to advance forest conversión in small pine plantations. Landscape and Urban Planning, 87: 201 - 209.

Varas C. 2000. Un proceso de desarrollo sostenible: El caso de Cullpe. Tierra Nuestra, Año 8 (7): 103 - 113. 